\title{
Review Article \\ Cryptic Transcription and Early Termination in the Control of Gene Expression
}

\author{
Jessie Colin, ${ }^{1,2}$ Domenico Libri, ${ }^{1,2}$ and Odil Porrua ${ }^{1,2}$ \\ ${ }^{1}$ LEA Laboratory of Nuclear RNA Metabolism, Centre de Génétique Moléculaire (CNRS), UPR3404, 1 Avenue de la Terrasse, \\ 91190 Gif sur Yvette, France \\ ${ }^{2}$ Centre for mRNP Biogenesis and Metabolism, Aarhus University, C.F. Møllers Alle, Building 130, 8000 Aarhus C, Denmark
}

Correspondence should be addressed to Odil Porrua, porrua-f@cgm.cnrs-gif.fr

Received 20 June 2011; Accepted 30 August 2011

Academic Editor: Damien Hermand

Copyright (C) 2011 Jessie Colin et al. This is an open access article distributed under the Creative Commons Attribution License, which permits unrestricted use, distribution, and reproduction in any medium, provided the original work is properly cited.

\begin{abstract}
Recent studies on yeast transcriptome have revealed the presence of a large set of RNA polymerase II transcripts mapping to intergenic and antisense regions or overlapping canonical genes. Most of these ncRNAs (ncRNAs) are subject to termination by the Nrd1-dependent pathway and rapid degradation by the nuclear exosome and have been dubbed cryptic unstable transcripts (CUTs). CUTs are often considered as by-products of transcriptional noise, but in an increasing number of cases they play a central role in the control of gene expression. Regulatory mechanisms involving expression of a CUT are diverse and include attenuation, transcriptional interference, and alternative transcription start site choice. This review focuses on the impact of cryptic transcription on gene expression, describes the role of the Nrd1-complex as the main actor in preventing nonfunctional and potentially harmful transcription, and details a few systems where expression of a CUT has an essential regulatory function. We also summarize the most recent studies concerning other types of ncRNAs and their possible role in regulation.
\end{abstract}

\section{Introduction}

The development of new technologies in the field of transcriptome analysis has revealed an unexpected level of complexity in the eukaryotic transcription landscape. High-resolution techniques as tiling arrays and, more recently, RNA deep-sequencing have shown that a large proportion of transcripts are not associated to well-defined functional units as genes, rRNA, tRNA, and so forth, giving rise to the concepts of "pervasive" and "hidden" transcription $[1,2]$. Those transcripts are often rapidly degraded, so that they remain invisible unless RNA degradation is prevented, for example, by inactivation of the degradation machinery.

Recent deep sequencing of nascent transcripts [3] has allowed a more direct analysis of RNA polymerase distribution in wild-type yeast cells obviating the need for working in mutants of the degradation pathway. These experiments have nicely confirmed the existence of hidden transcription.

In the yeast Saccharomyces cerevisiae, the main class of non-coding unstable RNAs transcribed by the RNA polymerase II is constituted by the Cryptic Unstable Transcripts
(CUTs). CUTs are capped, relatively small, with an average length of 200 to $500 \mathrm{bp}$ and with heterogeneous $3^{\prime}$ ends [4]. Their transcription is terminated by a pathway dependent upon the Nrd1 complex (see below), which targets them for polyadenylation and degradation by the TRAMP complex and the nuclear exosome, respectively $[5,6]$.

Work from two independent groups has provided a detailed picture of the genomic distribution of CUTs $[7,8]$, showing that the vast majority of these transcripts originate from nucleosome-free regions (NFRs) corresponding to promoter regions of bona fide genes. Importantly, most of the identified CUTs are transcribed divergently from the promoter region of annotated genes, suggesting that yeast promoters are intrinsically bidirectional. At least two different mechanisms control the intrinsic bidirectionality of promoters, which is a potential source of interference in gene expression. The first acts at the level of chromatin structure and involves different protein complexes implicated in modification of histones or chromatin remodelling that minimize spurious initiation $[3,9]$. The second is the Nrd1-dependent termination pathway, mentioned above, which provokes 
early termination and degradation of the transcripts (Figure 1). In addition to the more frequent antisense CUTs, a non-negligible number of CUTs overlap genes that are transcribed in the same sense. Some of these CUTs have been involved in the regulation of their cognate genes [ 7 , 10-12]. Therefore, CUTs can be by-products of divergent transcription, but also functional units with an important role in the control of gene expression.

Another abundant class of non-coding RNAs has been named SUTs for Stable Unannotated Transcripts [8]. Their origin is the same as for the CUTs ( $5^{\prime}$ and $3^{\prime}$ end NFRs), but it has been proposed that they differ in their transcription termination mode since they are stable, and thus detectable in wild-type conditions, and often longer than CUTs [1]. However this aspect remains elusive because inactivation of a component of the canonical mRNA termination pathway that depends on the CPF-CF complex (Cleavage and Polyadenylation Factor- Cleavage Factor I) has only a minor effect on the termination of the SUTs tested [13]. A regulatory role for at least a subset of SUTs has also recently been described [14]. Finally, a new category of ncRNAs has been described very recently, which includes mainly antisense transcripts that are stabilized upon mutation of the major cytoplasmic $5^{\prime}$ to $3^{\prime}$ exoribonuclease Xrn1p [15]. These ncRNAs have been designed as XUTs for Xrn1-sensitive Unannotated Transcripts. It has to be noted that often the distinction between XUTs and other ncRNAs is blurry as considerable overlap exists between these three classes of transcripts $[7,8]$. A role in repression of gene expression has been proposed for a subset of XUTs and their impact on transcription seems to be more prominent under stress conditions.

Because CUTs are by far the best-characterized class of ncRNAs in terms of origin, metabolism, and implication in regulation of gene expression, in this review we focus on the Nrd1-dependent termination pathway and its key role in limiting pervasive transcription and we describe the mechanisms of regulation that involve expression of a CUT. We also detail other cases of regulation mediated by ncRNAs belonging to other categories as SUTs or XUTs. The impact of cryptic transcription on global gene expression as well as the possible biological significance of this special way of regulation will be discussed.

\section{Early Termination and Degradation in the Control of Cryptic Transcription}

Pervasive transcription constitutes a risk for the cell that is controlled at different levels. Translation of aberrant or defective mRNAs that could result in toxic proteins is prevented by the nonsense-mediated decay (NMD), nonstop decay (NSD), and no-go decay (NGD) pathways in the cytoplasm [16]. However, those pathways act late in the expression process and cannot preclude possible interference of cryptic transcription with normal transcription of genes as, for example, by impeding binding of activator proteins to the promoter region of a downstream gene or by collision with elongating polymerases on a convergent gene [17-19]. Some of these deleterious effects are circumvented by the action of the
Nrd1-complex that simultaneously elicits termination early in transcription and recruits the exosome-TRAMP complexes to their target RNAs, facilitating polyadenylation and consequential degradation of the transcripts [20]. The exosome is a conserved multisubunit complex with both endonuclease and $3^{\prime}-5^{\prime}$ exonuclease activities that functions in degradation of defective transcripts as well as in processing of $3^{\prime}$ ends of stable ncRNAs (snRNAs, snoRNAs, and the $5.8 \mathrm{~S}$ rRNA). The exosome has a nuclear and a cytoplasmic forms that share a core of ten proteins, being Rrp44p (also named Dis3p) the sole catalytic subunit of the core exosome. The nuclear form of the exosome possesses an additional exonuclease, Rrp6p, that also partakes in the degradation of CUTs (reviewed in [16]). The TRAMP complex has two alternative forms with a common structure, containing a poly-A polymerase (either Trf4p or Trf5p, Trf4p-containing complexes being more abundant), the DexH-box helicase Mtr4p and a zinc-knuckle RNA binding protein (either Airlp or Air2p). In contrast to the protective role of poly-A tails in mRNAs, polyadenylation by the TRAMP complex promotes exosomemediated degradation, which is thought to be due to the lower processivity of $\operatorname{Tr} 4 / 5$, compared to the mRNA poly-A polymerase [21] and/or the shorter length of the poly-A tails added by the TRAMP complex [22]. The coupled action of the Nrd1-termination complex and the nuclear exosome allows controlling the production of aberrant transcripts at a stage prior to RNA export, possibly avoiding flooding the downstream RNA quality pathways mentioned above (NMD, NGD, etc.).

Even though the TRAMP complex and the nuclear exosome are important actors in the control of pervasive transcription, their role has been extensively reviewed elsewhere [23]. We will focus here on the properties of the Nrd1-complex and the data that contribute to elucidate the mechanisms of termination.

\section{The Nrd1-Nab3-Sen1 Termination Complex}

The Nrd1 complex was first identified for its role in termination and exosome-mediated maturation of sn- and snoRNAs [24]. Subsequently, it was shown to be responsible for termination of the novel class of ncRNAs designed as CUTs [5, $6]$.

The Nrd1 complex is composed by the RNA binding proteins Nrd1p and Nab3p and the RNA and RNA-DNA helicase Sen $1 \mathrm{p}$. Nrd1p is an essential $63 \mathrm{kDa}$ protein that contains an Nab3p interacting region, an RNA recognition motif (RRM) and an N-terminal region that allows interaction with the C-terminal domain (CTD) of the large subunit of RNAP II (CID, CTD Interacting Domain $[25,26]$ ). The CTD of RNAP II consists of tandem repeats of a hepta-peptide (YSPTSPS) that is subjected to different post-translational modifications throughout the transcription cycle and that serves as a landing pad for many proteins involved in key processes such as capping, elongation, termination, and splicing [27]. Phosphorylation at serines 2,5 , and 7 has been shown to be critical for the function and shape of the CTD. Nrdlp interacts preferentially with the Ser5-P form of RNAP II CTD in vitro 


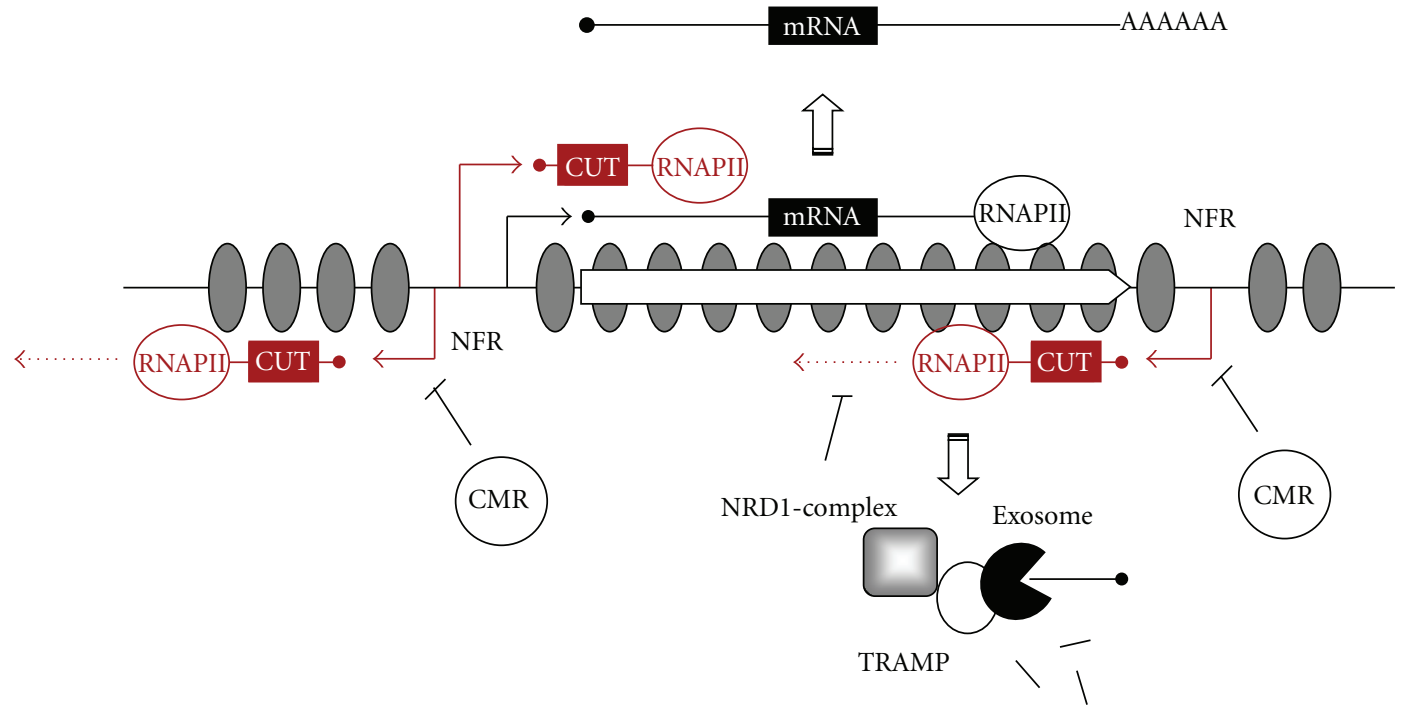

FIGURE 1: Complexity of the transcriptional landscape in yeast. Transcription of genes encoding stable RNAs by RNAP II is indicated by black lines and cryptic transcription originated from $5^{\prime}$ and $3^{\prime}$ nucleosome free regions (NFRs) is depicted in red. Initiation of transcription is represented by bent arrows and nucleosomes are depicted by grey ovals. Transcription can lead to the production of polyadenylated mRNAs that are competent for export to the cytoplasm and subsequent translation. Initiation of cryptic transcription is minimized by chromatin modifying and remodelling complexes (CMRs) that impose a repressive structure on the chromatin. When those mechanisms are insufficient, the Nrd1 complex terminates transcription and recruits the TRAMP and exosome complexes, which leads to polyadenylation and degradation of the generated CUT.

but colocalizes genomewide with the Ser7-P form in vivo [26, 28], suggesting an important role of the latter modification. $\mathrm{Nab} 3 \mathrm{p}$ is an essential $90 \mathrm{kDa}$ protein that contains an $\mathrm{N}$-terminal domain rich in $\mathrm{D}$ and $\mathrm{E}$, a central RRM, and an essential P/Q-rich C-terminal domain of unknown function [29]. It interacts directly with Nrdlp and with Sen1p $[29,30]$. Mutational analysis of Nrd1-dependent termination substrates and RNA-binding assays performed with purified RRMs has led to identification of GUAA/G and UCUU as binding sites, respectively, for Nrd1p and Nab3p [31-33]. In addition, in vivo RNA-protein crosslinking experiments (CRAC) have recently shown that the preferred binding site for Nab3p in vivo is CTTG [22]. Sen $1 \mathrm{p}$ is an essential $252 \mathrm{kDa}$ protein with a role in termination of ncRNAs as well as several mRNAs [34] that also functions in DNA repair [35, 36]. The first 975 amino acids of Sen $1 \mathrm{p}$ are dispensable for growth but are involved in the interaction with RNAPII, the RNAse III-like endonuclease Rntlp, and the nucleotide excision repair endonuclease $\operatorname{Rad} 2 p$ [35], while the C-terminal half contains the essential helicase domain and a motif required for interaction with the Glc7p phosphatase, which dephosphorylates Sen1p in vitro [30].

Unlike the canonical mRNA termination pathway that depends on the CPF-CF complex and functions late in transcription, Nrd1-dependent termination is efficient only within a window of less than $1000 \mathrm{bp}$ after transcription initiation, where the RNAP II CTD is phosphorylated mainly at Ser5 and Ser7 [28, 37-40].

Although it has been shown that Ser5-P (and possibly Ser7-P) favors Nrd1-dependent termination while Ser2-P antagonizes it [37], the correlation between CTD phosphorylation and termination remains not fully elucidated and the correct levels of each modification appear to be crucial. Consistent with this notion, mutation of proteins involved in the modification of the CTD, such as the Ser2 phosphatase Fcplp [37], the Ser2 kinase Ctk1p [24], the Ser5 kinase Kin28p, the phosphatase Ssu72p [41, 42], and even the SerPro isomerase Ess1p [43] affects negatively Nrd1-dependent termination. Interestingly, moreover, the role of the CTD and its modifications in termination might not pertain directly to its interaction with $\mathrm{Nrd} 1 \mathrm{p}$ because a $n r d 1-\Delta \mathrm{CID}$ mutant does not exhibit any detectable termination defect [26].

In contrast to the interaction with the CTD, interaction with the RNA is strictly required for Nrd1-dependent termination $[24,26,29,37]$, although the abundance of the known Nrd1p and Nab3p recognition sites within the different termination substrates is highly variable, ranging from one to more than twelve sites $[5,10]$. This variability suggests that additional termination signals yet unidentified might exist. Indeed, recent results obtained in our laboratory have revealed new motifs involved in Nrd1-dependent termination as well as specific arrangement of sites that are required for the termination signals to be functional (Porrua et al., unpublished). The heterogeneity among termination substrates concerns not only the cis-acting elements but also the trans-acting factors involved in Nrd1-dependent termination. For example, mutations in the catalytic site of the prolyl isomerase Esslp provoke a defect in termination of a set of snoRNAs but termination of other snoRNAs and a large share of CUTs remain unaffected [43]. In addition, the poly A-binding protein Hrplp seems to be implicated in termination of some CUTs tested but not others [10]. Furthermore, mutation or deletion of genes encoding proteins involved in histone modification as the histone methyltransferase SET1 
and the histone deacetylase complex Rpd3L exacerbates the termination defects of NRD1 mutants at most of the CUTs and snoRNAs analyzed but not all [44]. This apparent complexity is maybe the reason why the precise mechanism of Nrd1-dependent termination remains largely not understood. Further work, especially with in vitro systems, is needed to fully understand the exact role of each of the proteins involved as well as to dissect the different steps leading to transcription termination.

\section{Cryptic Transcription in the Control of Gene Expression}

Most of the CUTs that function in the regulation of gene expression identified thus far are located upstream or overlap the regulated gene and in the sense orientation. Production of the regulatory CUT normally has a negative impact on transcription of the downstream gene and this effect is exerted in cis CUTs can share the TATA box and/or the transcriptional start sites with the regulated gene or can use their owns, implying different mechanisms by which regulation occurs. In this section, we review the best-characterized examples of CUTs implicated in regulation and we briefly comment other types of ncRNAs that also control the expression of genes by different mechanisms.

4.1. Regulation by Attenuation. The term "attenuation" refers to negative control by a CUT that shares both the TATA box and the transcriptional start sites (TSS) with the regulated gene. After transcription initiation, a fraction of the elongating polymerases is subjected to early termination by the Nrd1-dependent pathway, which generates a non-functional CUT. Only the molecules that escape premature termination can proceed until the CPF-dependent terminator sequences, thus producing a functional mRNA molecule (Figure 2(a)).

The first and the best-characterized example of attenuation is autoregulation of NRD1 itself. The NRD1 transcript behaves both as an mRNA and as a CUT because it contains all the determinants for Nrd1-dependent termination and degradation, as well as the sequences required for normal termination and $3^{\prime}$ end formation by the canonical CPF-dependent pathway. The full-length NRD1 mRNA is upregulated upon mutation or inactivation of NRD1, Nab3p, or SEN1, $[24,45]$. Nrd1 has a relatively long $5^{\prime}$ UTR of about $300 \mathrm{bp}$ that contains Nrd1p and Nab3p binding sites. This sequence is sufficient for triggering Nrd1-dependent termination when inserted into an exogenous gene and provokes a 3- to4-fold reduction in the levels of NRD1 mRNA [24]. At least 13 additional Nrd1p- and Nab3p-binding sites are spread within the first $600 \mathrm{bp}$ of NRD1 coding sequence. Mutation of these motifs individually has a modest, if any, effect on NRD1 autoregulation, but mutation of all of them in conjunction with modification of the sites in the 5'UTR provokes an additional 2-fold increase of mRNA levels, indicating that sequences within the coding sequence contribute to autorepression [6].

Another gene that shares this mode of regulation is HRP1. As in the case of NRD1, the HRP1 5'UTR is sufficient to trigger early termination when inserted in an exogenous gene. Termination is impaired upon mutation of the components of the Nrd1-complex, but, interestingly, it is also negatively affected by mutation of HRP1 itself $[10,34]$. Hrp1p is an RNA-binding protein that interacts with AU dinucleotide repeats and is implicated in pre-mRNA cleavage and polyadenylation [46] and mRNA export from the nucleus [47]. The work of Kuehner and coauthors suggests that Hrp1p acts in concert with the Nrd1-complex to regulate its own expression and possibly the expression of other genes that are subjected to attenuation.

4.2. Regulation by Transcriptional Interference. One of the best examples of transcriptional interference that implicates the production of a CUT relates to the control of the SER3 gene, whose product catalyses a step in serine byosynthesis, in response to serine availability. The expression of SER3 is activated in the absence of serine and is repressed under serine-replete conditions by the expression of an upstream CUT designed as SER3 Regulatory Gene 1 (SRG1) [48]. SRG1 is transcribed from its own promoter and TSS, which are different from those of SER3, and it extends up to the first nucleotides of the SER 3 coding region.

In the presence of serine, the sequence-specific activator Cha4p recruits the Swi/Snf chromatin remodeler and the SAGA complex to SRG1 promoter region. These factors together activate transcription of SRG1 that subsequently impedes binding of transcription activators and the TATA-binding protein to the SER3 promoter region $[11,48]$. Recent results have shown that the mechanism of transcriptional interference involves the assembly of nucleosomes over the SER3 promoter region upon transcription of $S R G 1$, a process that is mediated by the action of Spt6p and Spt16p, two histone chaperones that facilitate disassembly and reassembly of nucleosomes [49]. In the absence of serine, Cha4p does not interact with Swi/Snf and SAGA complexes, so transcription activation of SRG1 does not take place and an NFR is formed at the SER3 promoter region, allowing the binding of sequence-specific activators that are required for full expression of SER3 (Figure 2(b)).

Termination of SRG1 transcription occurs at two consecutive sites, being termination at the first site dependent on the Nrd1-complex and termination at the second site presumably dependent on the CPF-pathway. Given that expression of SRG1 is strong and that each terminator alone might not be sufficiently efficient, the presence of a second terminator could constitute a fail-safe mechanism to prevent the production of a chimeric SRG1-SER3 transcript. Indeed, depletion of the Nrd1 leads to the accumulation of SRG1SER3 RNAs that might eventually give rise to functional Ser3p protein under repressing conditions or to aberrant, potentially toxic translation products [5].

A similar mechanism of transcriptional interference might operate on regulation of $A D H 1$ and $A D H 3$ expression in response to zinc-limitation. $A D H 1$ and $A D H 3$ encode two different zinc-dependent alcohol dehydrogenases that are repressed during zinc deficiency by the small upstream transcripts $Z R R 1$ and $Z R R 2$, respectively. Expression of 


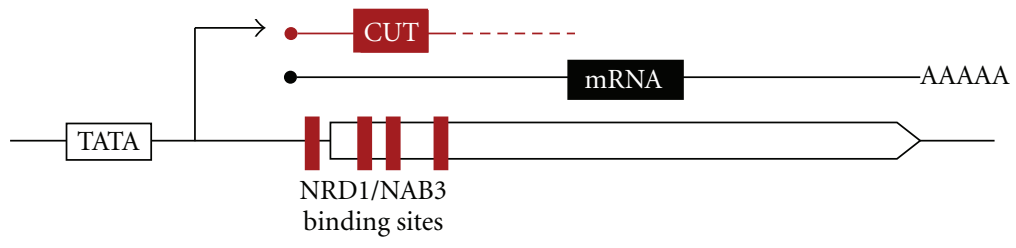

(a)

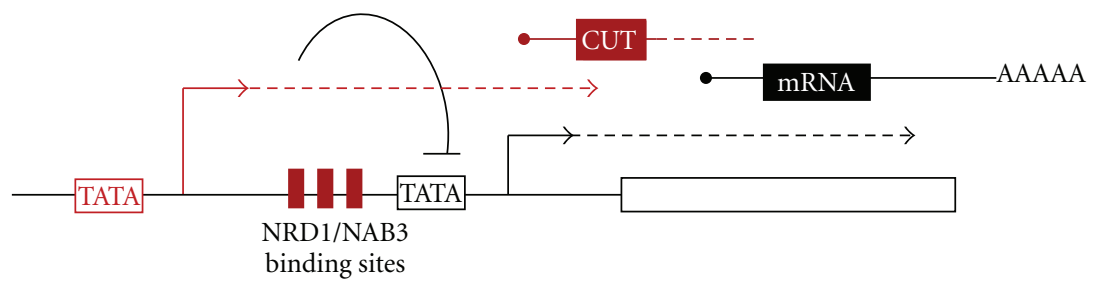

(b)
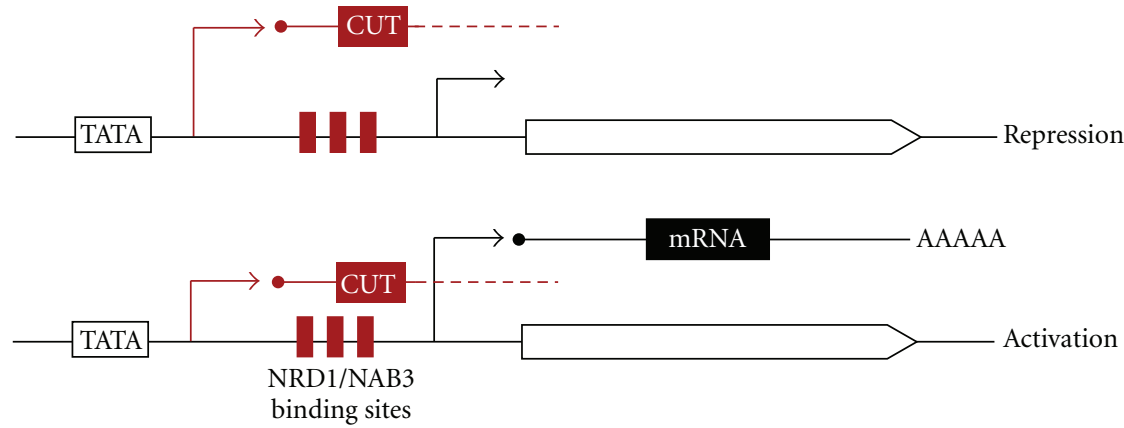

(c)

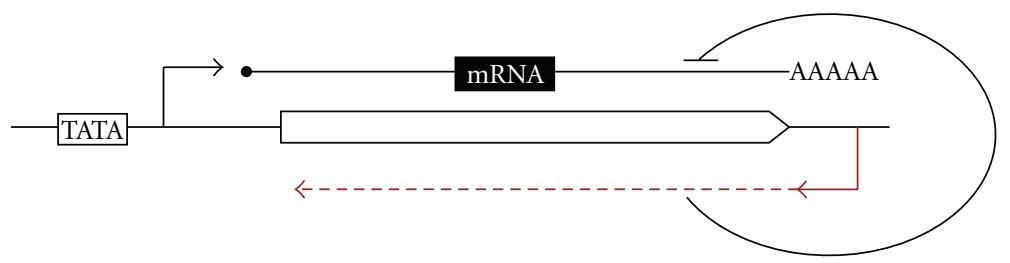

(d)

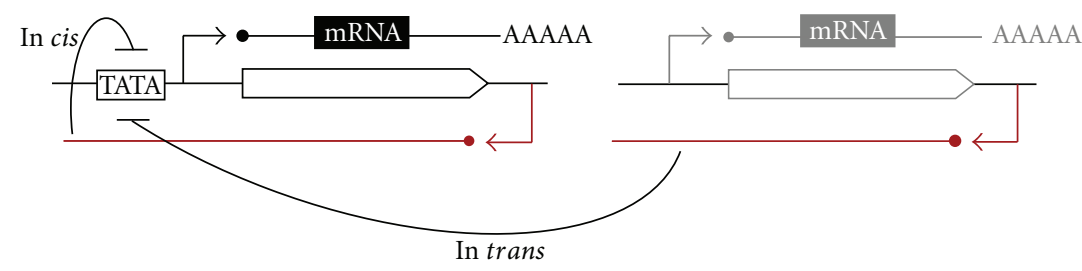

(e)

FIGURE 2: Summary of regulatory mechanisms involving production of ncRNAs Transcription is depicted by dashed arrows, ncRNAs by red lines, and mRNAs by black lines. Transcription start sites (TSSs) are indicated by bent arrows. Red boxes indicate Nrd1-dependent termination signals. (a) Regulation by attenuation: a given transcription initiation event can give rise to either an unstable transcript generated by premature termination by the Nrd1 complex or a stable mRNA if transcription is allowed to reach the CPF-dependent terminator. (b) Regulation by transcriptional interference: transcription of a CUT (or a stable RNA) occludes the promoter of a downstream mRNA gene thus impairing pre-initiation complex assembly and subsequent expression of the gene. (c) Regulation by alternative TSS choice: transcription initiation can occur either at an upstream or a downstream TSS. When the upstream TSS is selected, Nrd1-dependent termination signals are included in the transcript, leading to transcription termination and production of a CUT. When transcription starts at the downstream TSSs, these signals are skipped and a functional mRNA is produced. Regulation occurs at the level of TSS selection. (d) Regulation by antisense transcription: antisense transcription impairs the sense of mRNA production without affecting the initiation step. (e) Regulation by antisense $n c R N A$ : long ncRNAs are able to act in cis and in trans to recruit chromatin-modifying enzymes and silence the sense gene. In this case, regulation is mediated by the ncRNA. 
the upstream ncRNA is activated by the zinc-responsive regulator Zap1p, and transcription through the $A D H 1$ and $A D H 3$ promoter regions prevents binding of the required transcriptional activators [50]. Whether repression involves nucleosome deposition as for SER3 regulation remains to be assessed. Both transcripts are relatively stable and detectable in a wild-type strain, however, at least part of the termination might be Nrd1-dependent since they are recovered in the CUT fraction in deep sequencing transcriptome analysis [7].

Finally, strong expression of upstream sense CUTs has been detected at some genes involved in glycolysis as TPI1, GPM1, and FBA1. These CUTs are expressed from their own promoter and TSS and are antiregulated relative to their associated mRNA, suggesting that they might be subjected to the same mechanism of transcriptional interference as SER3 [7].

4.3. Alternative Transcriptional Start Site (TSS) Choice. Several genes of the nucleotides biosynthetic pathways URA2, IMD2, URA8, and ADE12, involved in synthesis of UTP, GTP, CTP, and ATP, respectively, are regulated by nucleotide availability and are activated when a given nucleotide is missing. All these loci share a similar organization with a $5^{\prime}$ overlapping CUT that is important for regulation (Figure 2(c)). The examples of IMD2 and URA2 are the best described $[10,12]$. In both cases, expression of the CUT and the mRNA is driven by a common promoter but transcription can start either at an upstream or a downstream site. The region between the two consecutive TSSs contains all the sequence elements required for Nrd1-dependent termination and degradation, so that the use of the upstream TSS leads to the production of a CUT. On the contrary, when transcription starts at the downstream site, the Nrd1 complex termination signals are skipped and the whole coding sequence can be transcribed, leading to the production of a functional mRNA. Selection of the mRNA TSS only occurs under activating conditions (i.e., shortage of the specific nucleotide) while only the CUT TSS is used under nucleotide replete conditions. However, since the common promoter is always active, most of the regulation occurs after preinitiation complex assembly at the step of start site selection.

The presence of the upstream TSS has a clear inhibitory effect on expression of the mRNA under nonactivating con ditions, due to the fact that most polymerases never make it to the downstream TSS. Indeed, it has been shown that mutation or deletion of the IMD2 CUT impairs full repression under guanine replete conditions [51]. Similarly, preventing production of the URA2 CUT by mutation of its start site leads to expression of URA2 mRNA even under nonactivating conditions [12].

What induces the selection of the downstream TSS upon activation remains, however, not fully clarified. It has been shown that under activating conditions the upstream CUT is still transcribed at the same levels as in repressing conditions in the case of URA2 and to somewhat lower levels in the case of IMD2. Therefore it remains an open question whether the selection of the mRNA TSS occurs because of increased read-through at the upstream CUT TSS or by some independent mechanism. Consistent with the first notion, it has been proposed that under GTP shortage, the upstream
TSS is skipped at the IMD2 locus because it involves a G as the starting nucleotide thus allowing initiation to the downstream mRNA start site [10]. However, regulation at URA2 has been shown to be different [12]. In this case, transcription of the CUT always starts at an A (not at U as the previous model would predict) and it is therefore difficult to imagine how UTP shortage could prevent initiation just based on nucleotide availability. Rather, it has been shown that activation of the mRNA sites requires a T-rich sequence comprised in the region between the CUT and the mRNA start sites and thus transcribed only at the CUT level [12]. This regulatory mechanism has revealed to be extremely complex and might be considerably different from one system to another. Additional work needs to be done on the different models to clarify the aspects that remain elusive. For example, it is unclear thus far whether, in addition to its repressive role under nonactivating conditions, expression of the upstream CUT plays any active role in selection of the downstream TSS upon nucleotide shortage [12]. However, it is clear that the Nrd1-dependent termination-degradation pathway plays an essential role in diverting the constitutive expression of a gene to a nonfunctional pathway, terminating and degrading transcripts initiated under conditions that do not require expression of the gene.

4.4. Other Ways of Regulation Involving an ncRNA. In this section, we include the most relevant cases of gene regulation that are mediated by other types of ncRNAs whose production is in principle independent of the Nrd1p pathway.

As we mentioned before, a second abundant class of ncRNAs includes stable transcripts that are originated by $5^{\prime}$ and $3^{\prime}$ NFRs as the CUTs and receive the name of SUTs. A recent genomewide study has shown that around $5 \%$ of genes overlap an SUT that is transcribed in the opposite strand and extends beyond the TSS of the gene. This set of ORFs is enriched in stress response and environment-specific genes and exhibits a larger expression dynamic range upon environmental changes, although identical maximal levels of expression, than genes with other configurations. The authors propose that in most cases, transcription of the antisense SUT represses expression of the sense gene under nonactivating conditions, which more efficiently locks the gene in an off-state under nonactivating conditions [14]. In addition to this possible general role of antisense transcription, condition-specific repression of a gene by an antisense ncRNA has been described in a number of cases. These sense-antisense modules can act through different mechanisms. They can act either in cis or in trans and several of them have been shown to be physiologically regulated as it is the case for the PHO84, IME4, ZIP2, or the GAL1-GAL10 loci.

IME4 and ZIP2 are two genes specifically expressed during meiosis and repressed in haploids by the cognate antisense ncRNAs RME2 and RME3, respectively $[18,52]$. These antisense transcripts provide a sophisticated way to activate genes as they are transcriptionally repressed in diploids by the a1p/ $\alpha 2 \mathrm{p}$ heterodimer, allowing expression of the meiotic genes. Control by RME2 and RME3 only works in cis and via a transcriptional interference-like mechanism. However, RME2 and RME3 ncRNAs do not need to extend until the 
promoter region of IME4 and ZIP2 to exert their inhibitory function and TBP is always present in the $5^{\prime}$ of the sense genes IME4 and ZIP2, even under repressive conditions. This suggests that the antisense RNA does not prevent formation of a preinitiation complex upstream of the sense gene but acts later, possibly by preventing elongation of the sense RNA (Figure 2(d)).

The GAL1-GAL10 locus provides another example of cisregulation by an ncRNA. At this locus, a transcript antisense to GAL10 is generated only in glucose while GAL1 and GAL10 are silent whereas it becomes undetectable in galactose media when GAL1 and GAL10 are active. This antisense RNA allows full repression of the GAL locus in low glucose conditions presumably by controlling the recruitment of chromatin remodelling enzymes such as the histone methyltransferase Set $1 p$ and the histone deacetylase complex Rpd3S $[53,54]$.

The PHO84 gene is also controlled by an antisense RNA. The ncRNA represses PHO84 transcription through different mechanisms as it acts not only in cis but also in trans and in both cases, production of a long antisense RNA spanning the PHO84 gene until its UAS sequence is necessary (Figure 2(e)). PHO84 is regulated in ageing cells, when the antisense RNA is stabilized thus turning off PHO84 expression. cis- antisense-mediated silencing requires recruitment of the histone deacetylase Hda1/2/3 which deacetylates histones at the PHO84 promoter locus [55]. The endogenous copy of $\mathrm{PHO} 84 \mathrm{can}$ also be silenced in trans by production of the antisense RNA from a plasmid, but in this case the silencing does not involve Hda1/2/3 [56].

The Ty1 transposon is also partially regulated by an antisense unstable transcript. This antisense RNA acts in trans to repress both mobility and expression of Ty1 [54]. The Ty1 antisense ncRNA is an XUT, and in a very recent work from van Dijk et al. [15], the authors propose that, as in the case of Tyl, many other genes are transcriptionally repressed by their antisense XUTs by a mechanism of silencing dependent on the action of the methyltransferase Setlp. Since repression is observed upon inactivation of the cytoplasmic RNAse Xrn1p, and subsequent stabilization of XUTs, an important mechanistic aspect that remains to be elucidated is how these cytoplasmic RNAs manage to exert their repressive role in the nucleus.

Additional data support a link between noncoding transcription and silencing $[57,58]$. However, in contrast to the regulatory systems mentioned above where an ncRNA promotes silencing, here stabilization of an ncRNA derived from heterochromatic rDNA spacer region counteracts silencing through modifications in the structure of chromatin. Nonetheless, whether this effect on silencing is mediated by transcription itself or by the ncRNA is still unclear.

Finally, a different example of regulation is that of the PHO5 locus, where transcription of an ncRNA antisense to PHO5 is necessary for nucleosome eviction at the promoter, which is required for transcriptional activation of the gene [59]. Thus, in this case the ncRNA plays a positive role in transcription, in contrast to the previously mentioned ncRNAs, which impact negatively the expression of the cognate genes.

\section{Concluding Remarks}

Cryptic transcription is widespread in yeast. Recent genomewide analyses of Saccharomyces cerevisiae transcriptome have revealed more than $1400 \mathrm{CUTs} / \mathrm{SUT}$ generated by NFRs, mainly at promoter regions of bona fide genes, either sense or antisense to the associated gene $[7,8]$. These studies have, however, been performed only in a few physiological conditions (exponential growth supported by a limited number of carbon sources) and upon mutation of only some of the components of the exosome and TRAMP complexes. Therefore, it is possible that many additional ncRNAs are produced under different conditions or in different growth phases. Consistently, a number of new unannotated transcripts have been detected upon deletion of the RNA exonuclease Xrn1p [15]. In addition, a recent analysis of the yeast transcriptome during sporulation has revealed the presence of new ncRNAs specific of the meiotic phase designed as MUTs for Meiotic Unannotated Transcripts [60]. Furthermore, several additional CUTs have been identified upon mutation of the catalytic subunit of the core exosome Dis3p (Gudipati et al., unpublished results). In addition to ncRNAs originated at $5^{\prime}$ and $3^{\prime}$ NFRs, cryptic transcription can occur at regions internal to genes upon mutation of the histone chaperones Spt6p and Spt16p [61], as well as the histone deacetylase complex Rpd3S and the histone methyltransferase Set2p [62], presumably because of the formation of transitory NFR in coding regions that can unveil cryptic promoters. This is in agreement with the notion that the chromatin structure plays an important role in the control of cryptic transcription.

The occurrence of such a high level of cryptic transcription might imply that transcription initiation by eukaryotic RNAPII can occur with relatively low specificity in the absence of active mechanisms to prevent it. This behavior is rather different from that of the prokaryotic RNA polymerase, which always binds motifs that are quite conserved and initiates transcription at a defined distance downstream from the promoter sequences [63]. In many ways, this intrinsic "promiscuity" of the eukaryotic RNAPII constitutes a disadvantage as it requires efficient pathways to (i) counteract the potentially deleterious effects of pervasive transcription on the expression of functional transcripts (e.g., the Nrd1-dependent termination pathway) and (ii) degrade a large amount of nonfunctional or aberrant RNAs that could impact negatively the physiology of the cell, for example, by sequestering the export and translation machineries or by producing toxic protein products upon translation (e.g., nuclear and cytoplasmic RNA quality control pathways). In spite of these drawbacks, the maintenance of such flexibility might imply some evolutionary and/or functional advantages. For instance, it is possible that the production of a plethora of different ncRNAs allows some of them to evolve towards functional molecules, which would then be stabilized and confer higher fitness to the cell. In addition, a high flexibility in transcription initiation provides multiple opportunities for regulation, enabling the development of sophisticated regulatory mechanisms as those reviewed here.

The expression of the ncRNA normally plays a repressive role on transcription of the cognate gene. In general, genes 
that are subjected to both positive and negative control have a higher dynamic range of expression, which allows a finetuned response to environmental conditions [14]. In addition, active repression under nonactivating conditions minimizes "leakyness" in gene expression, avoiding production of proteins when they are not required by the cell and/or waste of energy. A remaining question is why the relatively complex negative control involving production of an ncRNA should be more advantageous than a classical regulation system based on protein repressors. The answer is not always obvious. In the case of regulation by attenuation, where the Nrd1-complex elicits partial premature termination, this mechanism provides an efficient way for the proteins involved in termination to control their own production by a feedback loop. The mechanism of transcriptional interference might allow transcriptional activators to be turned into repressors using the same molecular mechanism by which they both promote expression of the target genes and transcription of an ncRNA that prevents expression of the repressed genes. Finally, the regulation by alternative TSS selection seems to be associated to particular metabolic pathways (i.e., nucleotides biosynthesis) that require a fast response to environmental changes because they lead to the production of key molecules whose shortage would impact many cellular processes. A promoter that is already activated but not physiologically functional because it leads to the production of a CUT is more susceptible to be diverted to a functional state in the presence of the appropriate signal because it is endowed with the proper chromatin structure and a repertory of transcription factors and RNAPIIs. In that sense, production of the CUT would enable a "preactivated" state of the promoter, so that it would be ready for a fast response to changes in the intracellular environment.

Among the hundreds of ncRNAs that are associated to bona fide genes, only a few have been studied thus far and it is likely that expression of many more is modulated by the presence of an ncRNA and that additional regulatory mechanisms exist. Much more experimental work is needed to evaluate the global impact of cryptic transcription on gene expression and to unveil the multiple associated mechanisms of regulation.

In conclusion, this paper is focused on the main progresses in the study of ncRNAs done in the model organism Saccharomyces cerevisiae over the last years. However, the phenomenon of cryptic transcription and the regulation of gene expression by ncRNAs are conserved from yeast to animals. The most relevant works concerning the characterization of the noncoding transcriptome and the multiple categories of ncRNAs, as well as their associated regulatory functions, present in higher eukaryotes are nicely detailed in other recent reviews $[1,2,64]$.

\section{Acknowledgments}

This work was supported by the CNRS, the ANR (Grant ANR-08-BLAN-0038-CSD 8), the Fondation pour la Recherche Médicale (FRM), and the Danish National Research Foundation. The research was carried out within the scope of the Associated European Laboratory (LEA) "Laboratory of Nuclear RNA Metabolism.” O. Porrua is supported by a fellowship from the European Molecular Biology Organization.

\section{References}

[1] A. Jacquier, "The complex eukaryotic transcriptome: unexpected pervasive transcription and novel small RNAs," Nature Reviews Genetics, vol. 10, no. 12, pp. 833-844, 2009.

[2] J. Berretta and A. Morillon, "Pervasive transcription constitutes a new level of eukaryotic genome regulation," EMBO Reports, vol. 10, no. 9, pp. 973-982, 2009.

[3] L. S. Churchman and J. S. Weissman, "Nascent transcript sequencing visualizes transcription at nucleotide resolution," $\mathrm{Na}$ ture, vol. 469, no. 7330, pp. 368-373, 2011.

[4] F. Wyers, M. Rougemaille, G. Badis et al., "Cryptic pol II transcripts are degraded by a nuclear quality control pathway involving a new poly(A) polymerase," Cell, vol. 121, no. 5, pp. 725-737, 2005.

[5] M. Thiebaut, E. Kisseleva-Romanova, M. Rougemaille, J. Boulay, and D. Libri, "Transcription termination and nuclear degradation of cryptic unstable transcripts: a role for the Nrd1-Nab3 pathway in genome surveillance," Molecular Cell, vol. 23, no. 6, pp. 853-864, 2006.

[6] J. T. Arigo, K. L. Carroll, J. M. Ames, and J. L. Corden, "Regulation of yeast NRD1 expression by premature transcription termination," Molecular Cell, vol. 21, no. 5, pp. 641-651, 2006.

[7] H. Neil, C. Malabat, Y. D’Aubenton-Carafa, Z. Xu, L. M. Steinmetz, and A. Jacquier, "Widespread bidirectional promoters are the major source of cryptic transcripts in yeast," Nature, vol. 457, no. 7232, pp. 1038-1042, 2009.

[8] Z. Xu, W. Wei, J. Gagneur et al., "Bidirectional promoters generate pervasive transcription in yeast," Nature, vol. 457, no. 7232, pp. 1033-1037, 2009.

[9] I. Whitehouse, O. J. Rando, J. Delrow, and T. Tsukiyama, "Chromatin remodelling at promoters suppresses antisense transcription," Nature, vol. 450, no. 7172, pp. 1031-1035, 2007.

[10] J. N. Kuehner and D. A. Brow, "Regulation of a eukaryotic gene by GTP-dependent start site selection and transcription attenuation," Molecular Cell, vol. 31, no. 2, pp. 201-211, 2008.

[11] J. A. Martens, P. Y. J. Wu, and F. Winston, "Regulation of an intergenic transcript controls adjacent gene transcription in Saccharomyces cerevisiae," Genes and Development, vol. 19, no. 22, pp. 2695-2704, 2005.

[12] M. Thiebaut, J. Colin, H. Neil et al., "Futile cycle of transcription initiation and termination modulates the response to nucleotide shortage in S. cerevisiae," Molecular cell, vol. 31, no. 5, pp. 671-682, 2008.

[13] S. Marquardt, D. Z. Hazelbaker, and S. Buratowski, "Distinct RNA degradation pathways and 3' extensions of yeast noncoding RNA species," Transcription, vol. 2, no. 3, pp. 145-154, 2011.

[14] Z. Xu, W. Wei, J. Gagneur et al., "Antisense expression increases gene expression variability and locus interdependency," Molecular Systems Biology, vol. 7, p. 468, 2011.

[15] E. L. van Dijk, C. L. Chen, Y. D’Aubenton-Carafa et al., "XUTs are a class of Xrn1-sensitive antisense regulatory non-coding RNA in yeast," Nature, vol. 475, no. 7354, pp. 114-117, 2011.

[16] J. Houseley, J. LaCava, and D. Tollervey, "RNA-quality control by the exosome," Nature Reviews Molecular Cell Biology, vol. 7, no. 7, pp. 529-539, 2006. 
[17] I. H. Greger, A. Aranda, and N. Proudfoot, "Balancing transcriptional interference and initiation on the GAL7 promoter of Saccharomyces cerevisiae," Proceedings of the National Academy of Sciences of the United States of America, vol. 97, no. 15, pp. 8415-8420, 2000.

[18] C. F. Hongay, P. L. Grisafi, T. Galitski, and G. R. Fink, "Antisense transcription controls cell fate in Saccharomyces cerevisiae," Cell, vol. 127, no. 4, pp. 735-745, 2006.

[19] A. C. Palmer, J. B. Egan, and K. E. Shearwin, "Transcriptional interference by RNA polymerase pausing and dislodgement of transcription factors," Transcription, vol. 2, no. 1, pp. 9-14, 2011.

[20] L. Vasiljeva and S. Buratowski, "Nrd1 interacts with the nuclear exosome for 3' processing of RNA polymerase II transcripts," Molecular Cell, vol. 21, no. 2, pp. 239-248, 2006.

[21] J. LaCava, J. Houseley, C. Saveanu et al., "RNA degradation by the exosome is promoted by a nuclear polyadenylation complex," Cell, vol. 121, no. 5, pp. 713-724, 2005.

[22] W. Wlotzka, G. Kudla, S. Granneman, and D. Tollervey, “The nuclear RNA polymerase II surveillance system targets polymerase III transcripts," EMBO Journal, vol. 30, no. 9, pp. 1790 $1803,2011$.

[23] J. Houseley and D. Tollervey, "The many pathways of RNA degradation," Cell, vol. 136, no. 4, pp. 763-776, 2009.

[24] E. J. Steinmetz, N. K. Conrad, D. A. Brow, and J. L. Corden, "RNA-binding protein Nrd1 directs poly(A)-independent 3'end formation of RNA polymerase II transcripts," Nature, vol. 413, no. 6853, pp. 327-331, 2001.

[25] N. K. Conrad, S. M. Wilson, E. J. Steinmetz et al., "A yeast heterogeneous nuclear ribonucleoprotein complex associated with RNA polymerase II," Genetics, vol. 154, no. 2, pp. 557$571,2000$.

[26] L. Vasiljeva, M. Kim, H. Mutschler, S. Buratowski, and A. Meinhart, "The Nrd1-Nab3-Sen1 termination complex interacts with the Ser5-phosphorylated RNA polymerase II C-terminal domain," Nature Structural and Molecular Biology, vol. 15, no. 8, pp. 795-804, 2008.

[27] S. Buratowski, "Progression through the RNA polymerase II CTD cycle," Molecular Cell, vol. 36, no. 4, pp. 541-546, 2009.

[28] H. Kim, B. Erickson, W. Luo et al., "Gene-specific RNA polymerase II phosphorylation and the CTD code," Nature Structural and Molecular Biology, vol. 17, no. 10, pp. 1279-1286, 2010.

[29] K. L. Carroll, R. Ghirlando, J. M. Ames, and J. L. Corden, "Interaction of yeast RNA-binding proteins Nrd1 and Nab3 with RNA polymerase II terminator elements," RNA, vol. 13, no. 3, pp. 361-373, 2007.

[30] E. Nedea, D. Nalbant, D. Xia et al., "The Glc7 phosphatase subunit of the cleavage and polyadenylation factor is essential for transcription termination on snoRNA genes," Molecular Cell, vol. 29, no. 5, pp. 577-587, 2008.

[31] K. L. Carroll, D. A. Pradhan, J. A. Granek, N. D. Clarke, and J. L. Corden, "Identification of cis elements directing termination of yeast nonpolyadenylated snoRNA transcripts," Molecular and Cellular Biology, vol. 24, no. 14, pp. 6241-6252, 2004.

[32] F. Hobor, R. Pergoli, K. Kubicek et al., "Recognition of transcription termination signal by the nuclear polyadenylated RNA-binding (NAB) 3 protein," Journal of Biological Chemistry, vol. 286, no. 5, pp. 3645-3657, 2011.

[33] B. M. Lunde, M. Hörner, and A. Meinhart, "Structural insights into cis element recognition of non-polyadenylated RNAs by the Nab3-RRM," Nucleic Acids Research, vol. 39, no. 1, pp. 337346, 2011.
[34] E. J. Steinmetz, C. L. Warren, J. N. Kuehner, B. Panbehi, A. Z. Ansari, and D. A. Brow, "Genome-wide distribution of yeast RNA polymerase II and its control by Sen 1 helicase," Molecular Cell, vol. 24, no. 5, pp. 735-746, 2006.

[35] D. Ursic, K. Chinchilla, J. S. Finkel, and M. R. Culbertson, "Multiple protein/protein and protein/RNA interactions suggest roles for yeast DNA/RNA helicase Sen1p in transcription, transcription-coupled DNA repair and RNA processing," Nucleic Acids Research, vol. 32, no. 8, pp. 2441-2452, 2004.

[36] H. E. Mischo, B. Gómez-González, P. Grzechnik et al., "Yeast Sen 1 helicase protects the genome from transcription-associated instability," Molecular Cell, vol. 41, no. 1, pp. 21-32, 2011.

[37] R. K. Gudipati, T. Villa, J. Boulay, and D. Libri, "Phosphorylation of the RNA polymerase II C-terminal domain dictates transcription termination choice," Nature Structural and Molecular Biology, vol. 15, no. 8, pp. 786-794, 2008.

[38] M. H. Jenks, T. W. O'Rourke, and D. Reines, "Properties of an intergenic terminator and start site switch that regulate IMD2 transcription in yeast," Molecular and Cellular Biology, vol. 28, no. 12, pp. 3883-3893, 2008.

[39] A. Mayer, M. Lidschreiber, M. Siebert, K. Leike, J. Söding, and P. Cramer, "Uniform transitions of the general RNA polymerase II transcription complex," Nature Structural and Molecular Biology, vol. 17, no. 10, pp. 1272-1278, 2010.

[40] J. R. Tietjen, D. W. Zhang, J. B. Rodríguez-Molina et al., "Chemical-genomic dissection of the CTD code," Nature Structural and Molecular Biology, vol. 17, no. 9, pp. 1154-1161, 2010.

[41] C. Ganem, F. Devaux, C. Torchet et al., "Ssu72 is a phosphatase essential for transcription termination of snoRNAs and specific mRNAs in yeast," EMBO Journal, vol. 22, no. 7, pp. 15881598, 2003.

[42] E. J. Steinmetz and D. A. Brow, "Ssu72 protein mediates both poly(A)-coupled and poly(A)-independent termination of RNA polymerase II transcription," Molecular and Cellular Biology, vol. 23, no. 18, pp. 6339-6349, 2003.

[43] N. Singh, Z. Ma, T. Gemmill et al., "The Ess1 prolyl isomerase is required for transcription termination of small noncoding RNAs via the Nrd1 pathway," Molecular Cell, vol. 36, no. 2, pp. 255-266, 2009.

[44] N. Terzi, L. S. Churchman, L. Vasiljeva, J. Weissman, and S. Buratowski, "H3K4 trimethylation by Set1 promotes efficient termination by the Nrd1-Nab3-Sen1 pathway," Molecular and Cellular Biology, vol. 31, pp. 3569-3583, 2011.

[45] E. J. Steinmetz, S. B. H. Ng, J. P. Cloute, and D. A. Brow, "Cisand trans-acting determinants of transcription termination by yeast RNA polymerase II," Molecular and Cellular Biology, vol. 26, no. 7, pp. 2688-2696, 2006.

[46] L. Minvielle-Sebastia, K. Beyer, A. M. Krecic, R. E. Hector, M. S. Swanson, and W. Keller, "Control of cleavage site selection during mRNA 3' end formation by a yeast hnRNP," EMBO Journal, vol. 17, no. 24, pp. 7454-7468, 1998.

[47] M. M. Kessler, M. F. Henry, E. Shen et al., "Hrp1, a sequence-specific RNA-binding protein that shuttles between the nucleus and the cytoplasm, is required for mRNA 3'-end formation in yeast," Genes and Development, vol. 11, no. 19, pp. 2545-2556, 1997.

[48] J. A. Martens, L. Laprade, and F. Winston, "Intergenic transcription is required to repress the Saccharomyces cerevisiae SER3 gene," Nature, vol. 429, no. 6991, pp. 571-574, 2004.

[49] S. J. Hainer, J. A. Pruneski, R. D. Mitchell, R. M. Monteverde, and J. A. Martens, "Intergenic transcription causes repression 
by directing nucleosome assembly," Genes and Development, vol. 25 , no. 1 , pp. 29-40, 2011.

[50] A. J. Bird, M. Gordon, D. J. Eide, and D. R. Winge, "Repression of $\mathrm{ADH} 1$ and $\mathrm{ADH} 3$ during zinc deficiency by Zap1-induced intergenic RNA transcripts," EMBO Journal, vol. 25, no. 24, pp. 5726-5734, 2006.

[51] K. A. Kopcewicz, T. W. O’Rourke, and D. Reines, "Metabolic regulation of IMD2 transcription and an unusual DNA element that generates short transcripts," Molecular and Cellular Biology, vol. 27, no. 8, pp. 2821-2829, 2007.

[52] B. Gelfand, J. Mead, A. Bruning et al., "Regulated antisense transcription controls expression of cell-type-specific genes in yeast," Molecular and Cellular Biology, vol. 31, no. 8, pp. 17011709, 2011.

[53] M. Pinskaya, S. Gourvennec, and A. Morillon, "H3 lysine 4 diand tri-methylation deposited by cryptic transcription attenuates promoter activation," EMBO Journal, vol. 28, no. 12, pp. 1697-1707, 2009.

[54] J. Houseley, L. Rubbi, M. Grunstein, D. Tollervey, and M. Vogelauer, "A ncRNA modulates histone modification and mRNA induction in the yeast GAL gene cluster," Molecular Cell, vol. 32, no. 5, pp. 685-695, 2008.

[55] J. Camblong, N. Iglesias, C. Fickentscher, G. Dieppois, and F. Stutz, "Antisense RNA stabilization induces transcriptional gene silencing via histone deacetylation in S. cerevisiae," Cell, vol. 131, no. 4, pp. 706-717, 2007.

[56] J. Camblong, N. Beyrouthy, E. Guffanti, G. Schlaepfer, L. M. Steinmetz, and F. Stutz, "Trans-acting antisense RNAs mediate transcriptional gene cosuppression in S. cerevisiae," Genes and Development, vol. 23, no. 13, pp. 1534-1545, 2009.

[57] J. Houseley, K. Kotovic, A. El Hage, and D. Tollervey, "Trf4 targets ncRNAs from telomeric and rDNA spacer regions and functions in rDNA copy number control," EMBO Journal, vol. 26, no. 24, pp. 4996-5006, 2007.

[58] L. Vasiljeva, M. Kim, N. Terzi, L. M. Soares, and S. Buratowski, "Transcription termination and RNA degradation contribute to silencing of RNA polymerase II transcription within heterochromatin," Molecular Cell, vol. 29, no. 3, pp. 313-323, 2008.

[59] J. P. Uhler, C. Hertel, and J. Q. Svejstrup, "A role for noncoding transcription in activation of the yeast PHO5 gene," Proceedings of the National Academy of Sciences of the United States of America, vol. 104, no. 19, pp. 8011-8016, 2007.

[60] A. Lardenois, Y. Liu, T. Walther et al., "Execution of the meiotic noncoding RNA expression program and the onset of gametogenesis in yeast require the conserved exosome subunit Rrp6," Proceedings of the National Academy of Sciences of the United States of America, vol. 108, no. 3, pp. 1058-1063, 2011.

[61] C. D. Kaplan, L. Laprade, and F. Winston, "Transcription elongation factors repress transcription initiation from cryptic sites," Science, vol. 301, no. 5636, pp. 1096-1099, 2003.

[62] M. J. Carrozza, B. Li, L. Florens et al., "Histone H3 methylation by Set 2 directs deacetylation of coding regions by $\mathrm{Rpd} 3 \mathrm{~S}$ to suppress spurious intragenic transcription," Cell, vol. 123, no. 4, pp. 581-592, 2005.

[63] T. Ghosh, D. Bose, and X. Zhang, "Mechanisms for activating bacterial RNA polymerase," FEMS Microbiology Reviews, vol. 34, no. 5, pp. 611-627, 2010.

[64] M. Wery, M. Kwapisz, A. Morillon et al., "Noncoding RNAs in gene regulation," Wiley Interdisciplinary Reviews: Systems Biology and Medicine, vol. 3, no. 6, pp. 728-738, 2011. 

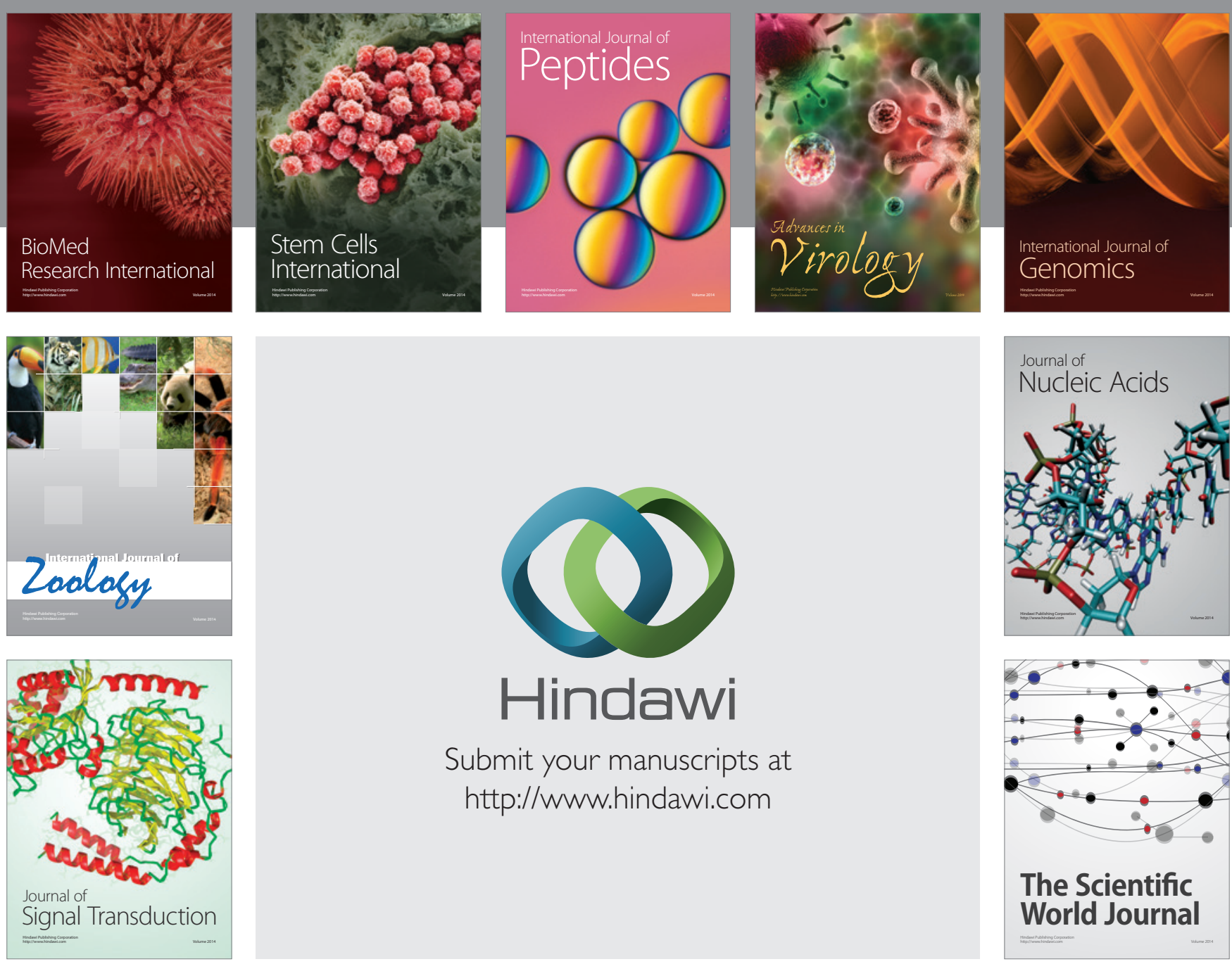

Submit your manuscripts at

http://www.hindawi.com
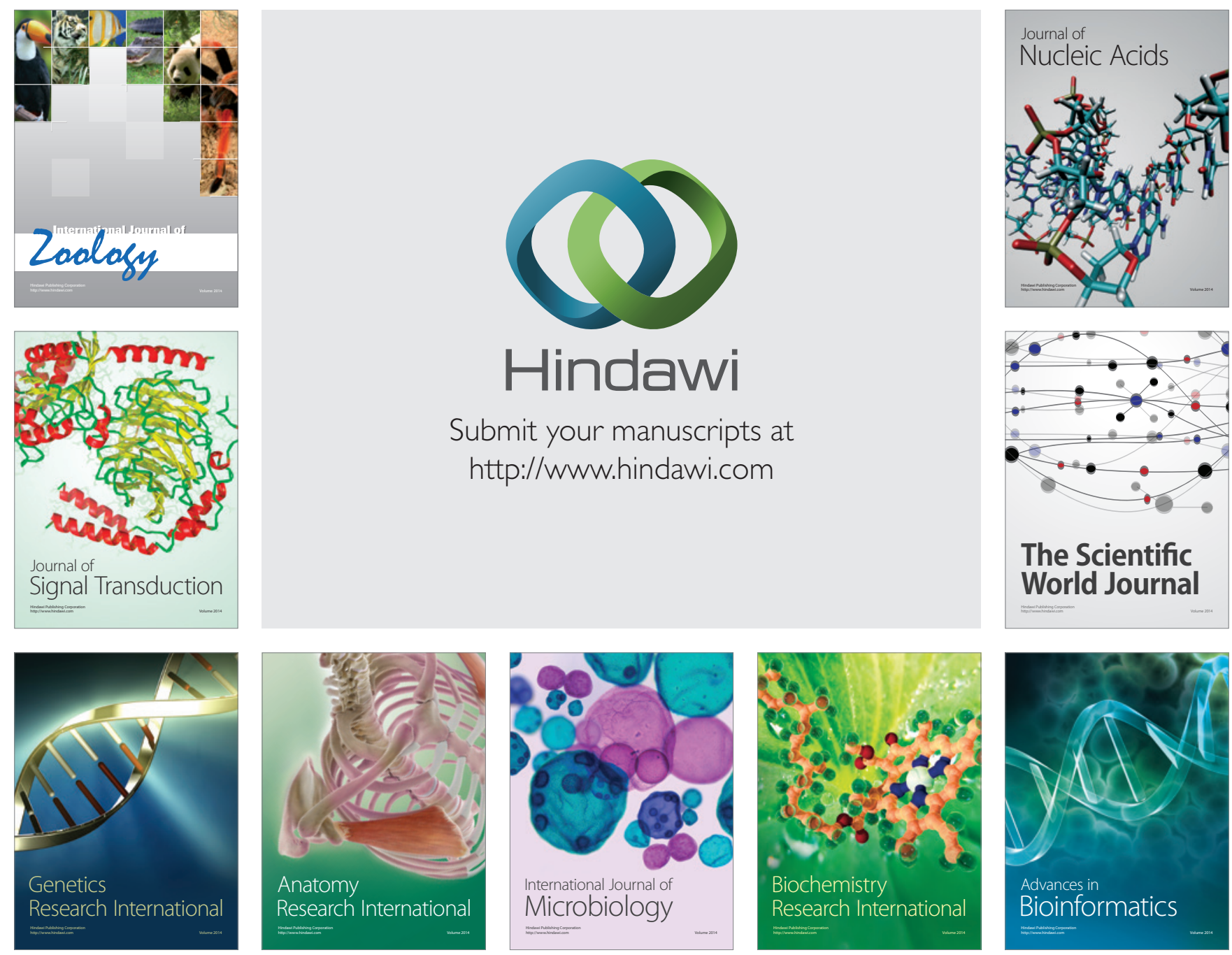

The Scientific World Journal
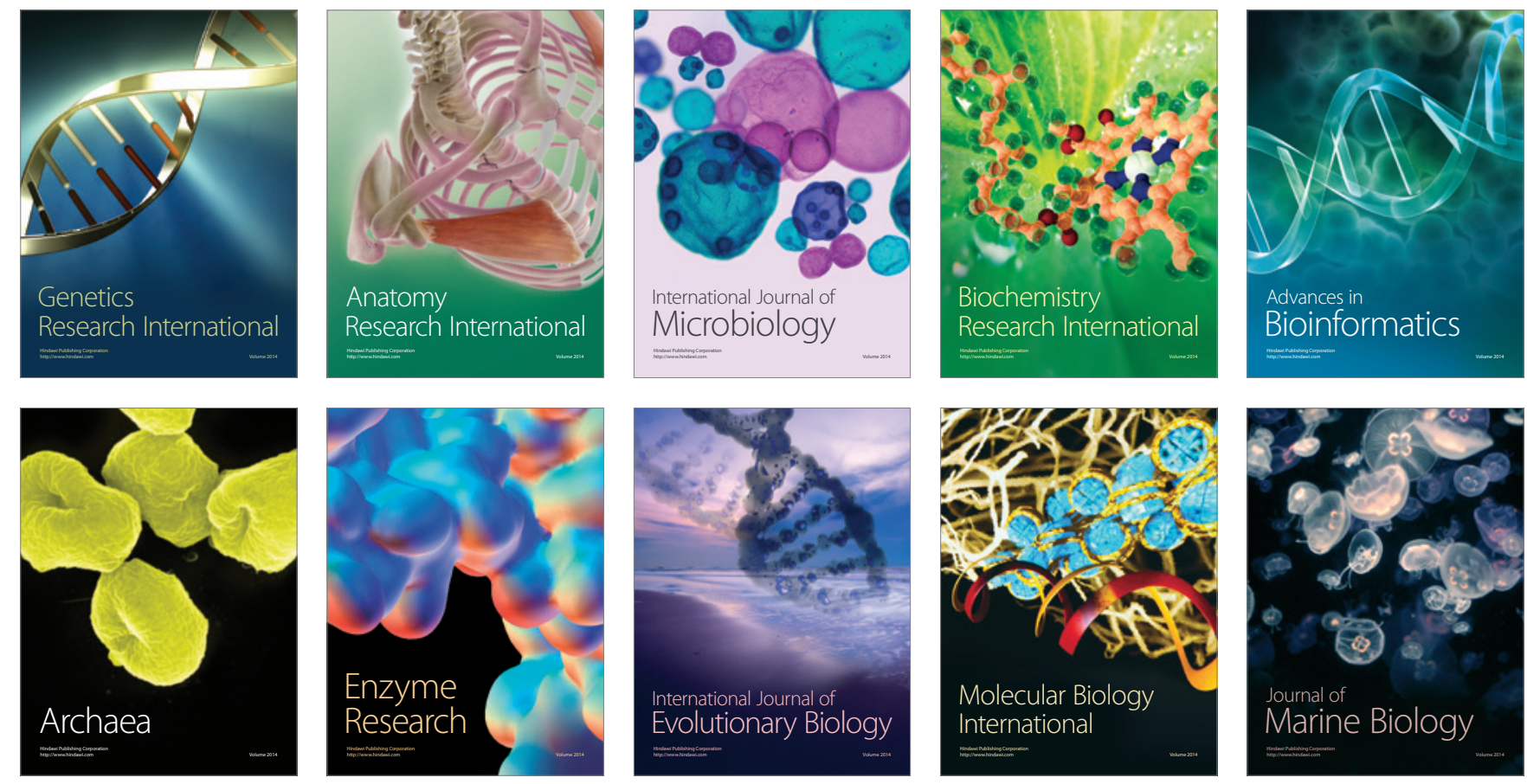